\title{
Ultrasensitive on-column detection of capillary electrophoresis using laser-induced capillary vibration method
}

\author{
T. Odake, T. Kitamori and T. Sawada
}

Department of Applied Chemistry, School of Engineering, The University of Tokyo, 7-3-1 Hongo, Bunkyo, Tokyo 113-8656, Japan

The laser-induced capillary vibration detection method, which the authors proposed and developed as a highly sensitive on-column detection method for capillary electrophoresis, is reviewed along with its applications to highly sensitive detection of biological materials.

apillary electrophoresis (CE), with its advantage of high resolution power, has attracted much attention.

But CE has another advantage of needing only a small sample volume, which is expected to make it a useful tool for ultramicroscale analysis as well. However, conventional absorbance detectors are not sensitive enough because the optical light-path is that of the diameter of a capillary, which is several tens of microns. A highly sensitive detector is indispensable to application of CE to ultramicroscale analysis. Several sensitive detection methods have been developed, such as laser-induced fluorometry (LIF), electrochemical detection, mass spectrometry, etc. To make the best use of the high separation efficiency of CE, an on-column detection scheme, which can easily reduce the detection volume needed by using a focused laser beam as a probing beam, seems to be more preferable than other detection schemes including off-column or end-column detection schemes, and LIF seems to be more suitable than electrochemical detection and mass spectrometric detection. However, LIF cannot be applied to nonfluorescent analytes, and fluorescent derivatization is usually needed, which is not suitable for ultramicroscale analysis.
Laser-induced photothermal spectrometry is another highly sensitive on-column detection approach, and differing from LIF, nonfluorescent species are also detectable. As a kind of photothermal effect, we found the capillary vibration induced by laser (CVL) effect [1], and proposed it as a highly sensitive on-column detection method of CE [2]. Like other photothermal detection methods, the CVL method is based on light absorption by a sample and successive heat generation due to nonradiative relaxation. This heat changes the capillary tension. Because the excitation laser beam is periodically irradiated onto the capillary, the tension fluctuation occurs periodically, resulting in vibration of the capillary. The amplitude of the capillary vibration has been found to be proportional to sample concentration. Therefore, quantitative analysis can be performed by detecting the amplitude of the capillary vibration.

We proposed a highly sensitive on-column detection of CE using the CVL effect. In this paper, we review the method and cite some applications.

\section{Generation mechanism of capillary vibration}

The CVL effect is a kind of photothermal effect caused by light absorption by a sample. Molecules excited by light relax by emitting energy in some form, which includes nonradiative relaxation and radiative relaxation. Substances which follow the latter process, for example, emitting fluorescence, are very rare, and most substances relax due to nonradiative processes emitting heat, which induces various photothermal phenomena. Therefore, photothermal spectrometries have an advantage of much wider applicability than radiative spectrometries such as LIF. 
(a)

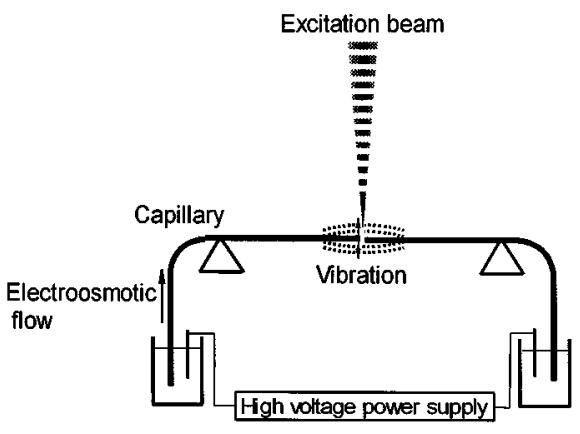

(b)

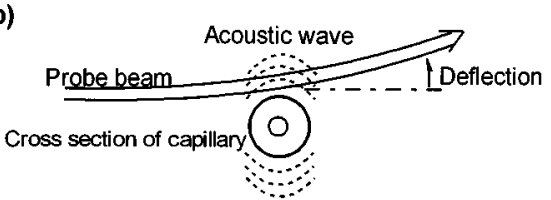

(c)

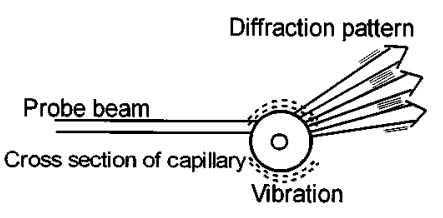

(d)

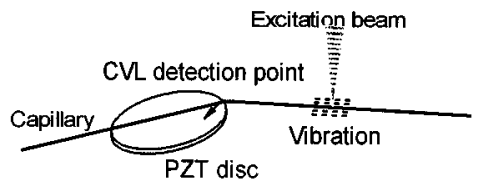

Figure 1. Experimental arrangement of $C E / C V L$ a), and detection methods of capillary vibration b-d). b) OBD method. c) Generation of diffraction pattern of a probe beam and direct detection of capillary vibration. d) Direct detection of capillary vibration using a PZT disc.

Generated heat in a capillary tube raises the temperature of the sample and the capillary tube itself. If the capillary is held tightly, the generated heat changes the tension of the capillary. If the excitation laser beam is intensity-modulated, capillary tension also fluctuates with the same period, which results in a string-like vibration of the capillary (Fig. 1a). The time constant of CVL is determined by heat dissipation time. From the thermal property of glass, heat dissipation time is calculated as around $1 \mathrm{~ms}$ [3]. Therefore, the excitation laser beam is modulated around $1 \mathrm{kHz}$ to obtain the best signal-to-noise ratio.

\section{Capillary vibration detection using an optical beam deflection method}

From our study on photoacoustic and photothermal phenomena, capillary vibration was deduced to emit an acoustic wave, which causes a change in refractive index of the surrounding air [2]. Therefore, a probe beam pass- (a)

(b)

(c)

(d)

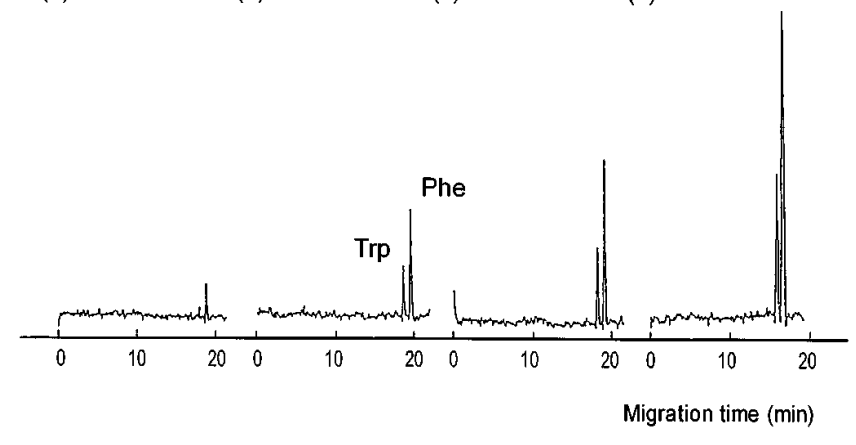

Figure 2. Sensitive and quantitative detection of nonderivatized amino acids by CE/CVL. Excitation laser was the second harmonic of an argon ion laser $(257 \mathrm{~nm}, 5 \mathrm{~mW})$. Injected amounts of Trp: a) $0.1 \mathrm{pmol}$, b) $0.3 \mathrm{pmol}$, c) $0.6 \mathrm{pmol}$, d) 1.2 pmol. Injected amounts of Phe: a) $1.0 \mathrm{pmol}$, b) $3.0 \mathrm{pmol}$, c) 6.0 pmol, d) 12 pmol. Capillary: $30 \mu \mathrm{m}$ i.d., $150 \mu \mathrm{m}$ o.d.

ing through the air very close to the vibrating capillary is deflected (optical beam deflection, OBD, Fig. 1b). Degree of deflection is proportional to sample concentration. Mixtures of tryptophan (Trp) and phenylalanine (Phe) solutions, introduced into a capillary were separated by $\mathrm{CE}$ and sensitively detected by the CVL method (Fig. 2). In this experiment, the second harmonic of an argon ion laser (257 nm, $5 \mathrm{~mW}$ ) was used as the CVL excitation source. Because Trp and Phe have absorptions at this wavelength, these amino acids can be detected without derivatization. The peak heights show good linearity for the injected amounts of the samples. By doubling the noise, the lower detection limit of absorbance is calculated to be about $8.0 \times 10^{-6}$, which is two or three orders of magnitude more sensitive than the conventional absorbance detector. Considering the CVL signal is proportional to the excitation beam power, this detection limit can be improved easily by using higher power UV lasers.

This OBD detection method, however, has a problem in adjusting the beam alignment, because the irradiation position of the probe beam is very critical. We developed a simple and easy detection method of capillary vibration, which does not involve energy conversion processes such as mechanical energy (vibration) to acoustic wave energy. This detection method was expected to be more effective to detect vibration energy and lead to more sensitive detection.

\section{Direct detection of capillary vibration}

The amplitude of capillary vibration is very small and considered to be proportional to the vibration energy, which is the conversion product of heat generated from the sample molecules. We developed a direct detection method of capillary vibration using a probe beam [4]. When a probe beam is passed through the capillary wall, a diffraction pattern is generated (Fig. 1c). The position of this pattern changes according to the vibration movement of the capillary. Therefore, by monitoring the vertical movement of the pattern using a position sensitive detector, the amplitude of 
the capillary can be detected. This detection method was proved to be at least one order of magnitude more sensitive than the OBD method, and alignment of the probe beam is easier because the probe beam position is checked visually by the diffraction pattern.

The vibration energy was also considered to be directly measured as stress applied to a piezoelectric transducer (PZT) disc (20 mm diameter, $2 \mathrm{~mm}$ thick), which was used as one of the supports of the vibrating capillary (Fig. 1d) [5]. In this detection system, sensitivity was also proved to be at least one order of magnitude higher than the OBD method.

Highly sensitive on-column detection of capillary electrophoresis was performed using the direct detection method of CVL. The sample was a mixture of dabsylized amino acids and an argon ion laser (488 nm, $100 \mathrm{~mW}$ ) was used as the CVL excitation source. Estimated injected amount of each amino acid was 15 fmol. Four amino acids were separated and sensitively detected (Fig. 3) [4].

\section{Generation of stationary wave CVL by pulsed laser excitation}

The CVL detection method can be applied to light-absorbing species. Therefore, to increase detectable species, it is effective to extend the choices of applicable lasers as a CVL excitation source. We have used a continuous wave (CW) laser as a CVL excitation source, with which we induced a forced vibration of the CVL. If we can induce the same stationary wave CVL using a pulsed laser, we can get a sensitive detection means for more kinds of species. However, a pulsed laser cannot usually induce a stationary wave, rather it leads to a damped wave. Since the peak height of the damped wave is also dependent on absorbance, quantitative analysis is possible. In this detection method, however, the peak height is easily affected by intensity fluctuation of each pulse so that baseline fluctuation prevents highly sensitive detection. We found a stationary wave could be induced by adjusting the repetition rate of the pulsed laser to the natural frequency of the capillary vibration. This technique is especially useful for detection of biological materials, because most biological materials have absorption bands in the UV region and a high performance pulsed UV laser has become available in recent years.

\section{Application to highly sensitive and direct detection of biological materials}

Using the above mentioned technique, amino acids, DNA fragments and many other biological materials are considered to be sensitively detected without derivatization by using a pulsed UV laser as a CVL excitation source. We introduced a waveguide $\mathrm{KrF}$ excimer laser $(248 \mathrm{~nm}$, $15 \mu \mathrm{J} /$ pulse) as a pulsed UV laser. Nonderivatized Trp and Phe, introduced and separated in a capillary, were detected using the stationary wave CVL technique (Fig. 4) [3]. Even in this pulsed laser excitation, the detection limit of

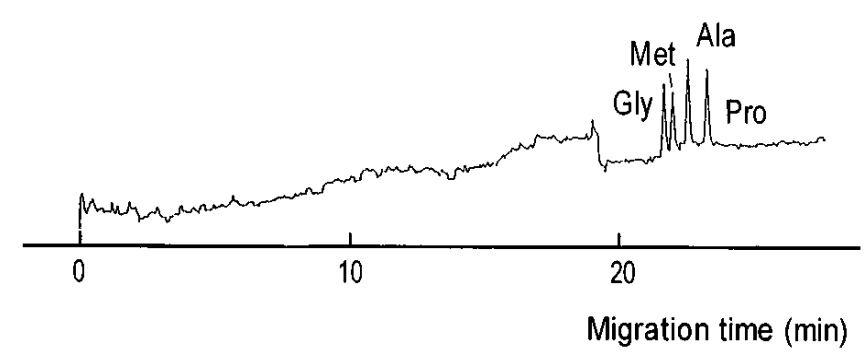

Figure 3. Highly sensitive detection of dabsylized amino acids by $C E / C V L$. Excitation laser was argon ion laser $(488 \mathrm{~nm}$, $100 \mathrm{~mW}$ ). Injected amount of each amino acid was $15 \mathrm{fmol}$. Capillary: $30 \mu \mathrm{m}$ i.d., $150 \mu \mathrm{m}$ o.d.

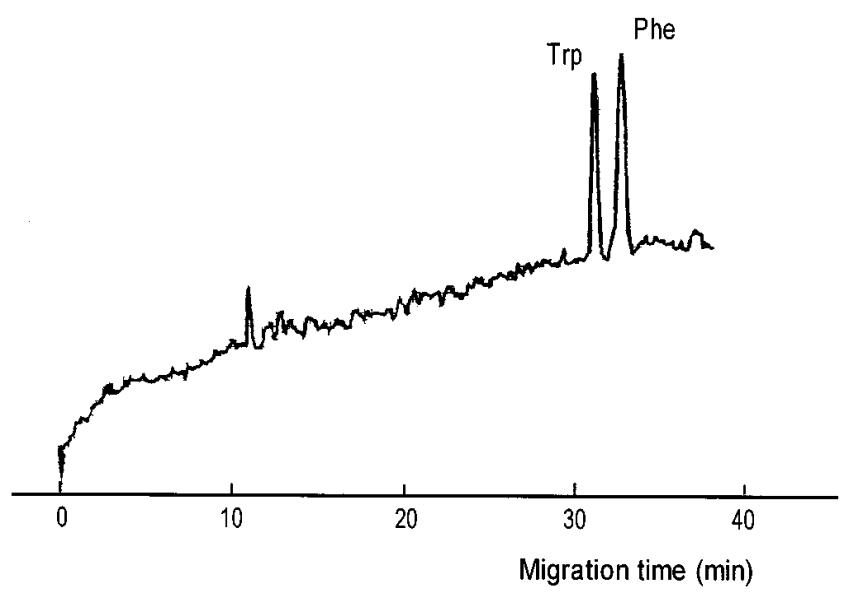

Figure 4. Highly sensitive and direct detection of nonderivatized amino acids by CE/CVL. Excitation laser was KrF excimer laser (248 $\mathrm{nm}, 10 \mu \mathrm{J} /$ pulse). Injected amounts of each amino acid were 0.3 pmol for Trp and 3.0 fmol for Phe. Capillary: $20 \mu \mathrm{m}$ i.d., $150 \mu \mathrm{m}$ o.d.

absorbance was almost the same as in continuous wave laser excitation.

It is also desirable to separate DNA fragments at high speed and sensitivity, and capillary gel electrophoresis has been shown to provide speedy separation of DNA fragments. The CVL detection method using a pulsed UV laser seems efficient for sensitive detection of DNA fragments without derivatization. However, another problem concerning the pulsed laser excitation occurs. The pulse radiation is so intense that sieving matrices, such as polyacrylamide gel, are easily destroyed. Considering that a separation medium with fluidity would avoid this destruction problem, we selected methylcellulose solution as a separation matrix for DNA fragments. The polymerase chain reaction (PCR) product $(500 \mathrm{bp})$ was separated by $\mathrm{CE}$ using the methylcellulose solution and detected without derivatization using the CVL method (Fig. 5) [6]. The detection limit of absolute amount was calculated to be 7 amol by doubling the signal-to-noise ratio. This result has almost the same sensitivity as LIF provides. 


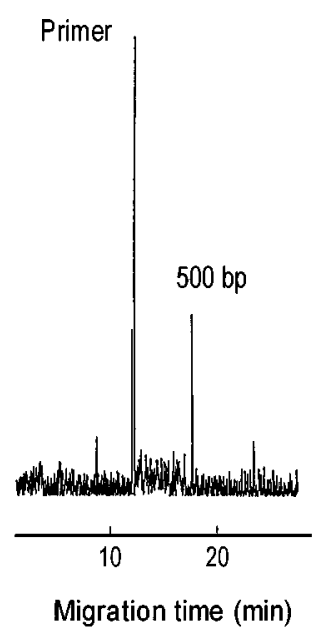

Figure 5. Highly sensitive and direct detection of PCR product by $\mathrm{CE} / \mathrm{CVL}$. Excitation laser was $\mathrm{KrF}$ excimer laser $(248 \mathrm{~nm}$, $10 \mu \mathrm{J} /$ pulse). Injection: $10 \mathrm{kV}, 3 \mathrm{~s}$. Separation: $10 \mathrm{kV}$. Capillary: $30 \mu \mathrm{m}$ i.d., $150 \mu \mathrm{m}$ o.d.

\section{Conclusion}

Development of highly sensitive on-column detection method using the CVL effect was reviewed. Both a CW laser and a pulsed laser were proved able to induce a stationary wave of CVL under certain conditions and provide highly sensitive detection. The CVL detection method was shown to have wide applicability, which suggested highly sensitive detection without derivatization. Highly sensitive on-column detections of amino acids and DNA fragments were demonstrated. These advantages of the CVL method are useful not only for practical applications such as gene diagnosis and DNA sequencing, but also for monitoring reactions at an ultramicroscale, such as on-column reactions and many metabolic processes. The CVL detection method is expected to be utilized for many kinds of such ultramicro and ultrasensitive analyses.

\section{References}

1. Wu, J.; Kitamori, T.; Sawada, T. Anal. Chem. 1990, 62, 1676.

2. Wu, J.; Odake, T.; Kitamori, T.; Sawada, T. Anal. Chem. 1991, 63, 2216.

3. Odake, T.; Kitamori, T.; Sawada, T. Anal. Chem. 1997, 69, 2537.

4. Odake, T.; Kitamori, T.; Sawada, T. Anal. Chem. 1995, 67, 145.

5. Odake, T.; Kitamori, T.; Sawada, T. Anal. Chem. 1992, 64, 2870.

6. Odake, T.; Sato, T.; Kitamori, T.; Sawada, T. Bunseki Kagaku 1997, 46, 415. 